\title{
Phase Transition in the Three-State Potts Antiferromagnet on the Diced Lattice
}

\author{
Roman Kotecký, ${ }^{1,2}$ Jesús Salas, ${ }^{3}$ and Alan D. Sokal ${ }^{4,5}$ \\ ${ }^{1}$ Center for Theoretical Study, Charles University, Prague, Czech Republic \\ ${ }^{2}$ Mathematics Institute, University of Warwick, Coventry CV4 7AL, United Kingdom \\ ${ }^{3}$ Gregorio Millán Institute, Universidad Carlos III de Madrid, 28911 Leganés, Spain \\ ${ }^{4}$ Department of Physics, New York University, 4 Washington Place, New York, New York 10003, USA \\ ${ }^{5}$ Department of Mathematics, University College London, London WC1E 6BT, United Kingdom
}

(Received 18 February 2008; revised manuscript received 22 April 2008; published 17 July 2008)

\begin{abstract}
We prove that the 3-state Potts antiferromagnet on the diced lattice (dual of the kagome lattice) has entropically driven long-range order at low temperatures (including zero). We then present Monte Carlo simulations, using a cluster algorithm, of the 3-state and 4-state models. The 3-state model has a phase transition to the high-temperature disordered phase at $v=e^{J}-1=-0.860599 \pm 0.000004$ that appears to be in the universality class of the 3-state Potts ferromagnet. The 4-state model is disordered throughout the physical region, including at zero temperature.
\end{abstract}

DOI: 10.1103/PhysRevLett.101.030601

PACS numbers: 64.60.Cn, 05.50.+q, 11.10.Kk, 64.60.F-

The $q$-state Potts model [1,2] plays an important role in the theory of critical phenomena, especially in two dimensions [3-5], and has applications to various condensedmatter systems [2]. Ferromagnetic Potts models are by now fairly well understood, thanks to universality; but the behavior of antiferromagnetic Potts models depends strongly on the microscopic lattice structure, so that many basic questions must be investigated case by case: Is there a phase transition at finite temperature, and if so, of what order? What is the nature of the low-temperature phase(s)? If there is a critical point, what are the critical exponents and the universality classes? Can these exponents be understood (for two-dimensional models) in terms of conformal field theory [5]?

One expects that for each lattice $\mathcal{L}$ there exists a value $q_{c}(\mathcal{L})$ such that for $q>q_{c}(\mathcal{L})$ the model has exponential decay of correlations uniformly at all temperatures, including zero temperature, while for $q=q_{c}(\mathcal{L})$ the model has a zero-temperature critical point. For $q<q_{c}(\mathcal{L})$ any behavior is possible; often (though not always) the model has a phase transition at nonzero temperature, which may be of either first or second order [6]. The first task, for any lattice, is thus to determine $q_{c}$.

Some two-dimensional antiferromagnetic models at zero temperature have the remarkable property that they can be mapped onto a "height" (or "interface" or "solidon-solid-type") model [8]. Experience tells us that when such a representation exists, the corresponding zerotemperature spin model is most often critical [9]. The long-distance behavior is then that of a massless Gaussian with some (a priori unknown) "stiffness" $K>$ 0 . The critical operators can be identified via the height mapping, and the corresponding critical exponents can be predicted in terms of the single parameter $K$. Height representations thus provide a means for recovering a sort of universality for some (but not all) antiferromagnetic mod- els and for understanding their critical behavior in terms of conformal field theory [5].

In particular, when the $q$-state zero-temperature Potts antiferromagnet on a two-dimensional lattice $\mathcal{L}$ admits a height representation, one expects that $q=q_{c}(\mathcal{L})$. This prediction is confirmed in all heretofore-studied cases: 3 -state square-lattice [8,10-12], 3-state kagome [13,14], 4-state triangular [15], and 4-state on the line graph (= covering lattice) of the square lattice $[14,16]$.

We now wish to observe that the height mapping employed for the 3-state Potts antiferromagnet on the square lattice [8] carries over unchanged to any planar lattice in which all the faces are quadrilaterals. One there-

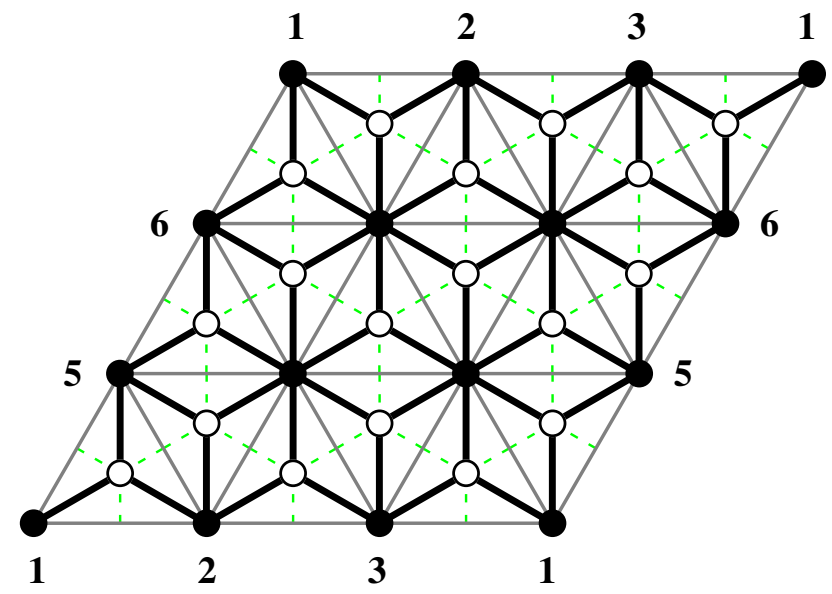

FIG. 1 (color online). A diced lattice of size $3 \times 3$ with periodic boundary conditions (edges depicted with thick black lines). The full circles show the sites of degree 6 , which form a triangular lattice (edges depicted with thin gray lines). The open circles show the sites of degree 3 , which form a hexagonal lattice (edges depicted with thin dashed green lines) that is the dual of the triangular lattice. Periodic boundary conditions are implemented by identifying border sites with the same label. 
fore expects that $q_{c}=3$ for every (periodic) plane quadrangulation.

The diced lattice (Fig. 1) is a periodic tiling of the plane by rhombi having $60^{\circ}$ and $120^{\circ}$ interior angles; in particular, it is a plane quadrangulation in which all vertices have degree 3 or 6 . The diced lattice is the dual of the kagome lattice, which is in turn the medial graph of the triangular and hexagonal lattices.

In this Letter we give a rigorous proof that the 3-state diced-lattice Potts antiferromagnet has a phase transition at nonzero temperature. This shows that, contrary to theoretical expectations, $q_{c}$ (diced) $>3$. It provides, moreover, the first example of a bipartite two-dimensional lattice in which $q_{c}>3$ (but see below).

Furthermore, this model provides a simple concrete example of entropically driven long-range order, in which coexistence between regions of different types of order on one sublattice is disfavored because it reduces the freedom of choice of spins on the other sublattice. Though this idea is intuitively appealing, it is difficult to determine, in any specific case, whether the mechanism is strong enough to produce long-range order. Here we are able to resolve this question rigorously by a conceptually simple though numerically delicate Peierls argument.

The existence (though not the nature) of a phase transition in this model is not, however, totally unexpected. A decade ago, Jensen et al. [17] computed low-temperature expansions for the 3-state and 4-state Potts models on the kagome lattice and found, among other things, indications of singularities in the unphysical region at $v=-3.486 \pm$ 0.003 and $v=-3.38 \pm 0.06$, respectively (here $v=e^{J}-$ 1 where $J$ is the nearest-neighbor coupling and we take $\beta=1$ ). Shortly thereafter, Feldmann et al. [18] used the duality $v \mapsto q / v$ of $q$-state Potts models on planar lattices to deduce predictions for the singularities of the 3-state and 4-state Potts models on the diced lattice: $v=-0.8607 \pm$ 0.0008 and $v=-1.18 \pm 0.02$, respectively. The latter occurs in the unphysical region at $v<-1$, suggesting that the 4-state diced-lattice antiferromagnet lies in a disordered phase at all temperatures, including zero temperature. The former, by contrast, lies within the physical antiferromagnetic regime at $J=-1.971 \pm 0.006$. If these predictions are correct, we have $3<q_{c}$ (diced) $<4$; crude linear interpolation suggests $q_{c}$ (diced) $\approx 3.4$.

One might, however, worry that the errors in the series extrapolation are radically larger than estimated and that the diced-lattice singularity lies not at $v \approx-0.86$ but instead at the theoretically expected $v=-1$ (which is not, after all, so far away). It is thus important to obtain independent evidence on the location of the phase transition (if any) in the 3-state diced-lattice Potts antiferromagnet. We do this in two steps: a mathematically rigorous proof of a phase transition at nonzero temperature, which furthermore elucidates the entropically driven nature of the corresponding long-range order; and Monte Carlo simula- tions to locate the phase transition and investigate its properties.

Proof of phase transition. - We shall prove that, at all sufficiently low temperatures, there is antiferromagnetic long-range order in which the spins on the triangular sublattice take preferentially one value and the spins on the hexagonal sublattice take more-or-less randomly the other two values. To implement the heuristic idea of entropically driven order, we evaluate the cost of coexistence between regions of unequal spins on the triangular sublattice by integrating out the spins on the hexagonal sublattice, yielding a $q$-state Potts model on the triangular lattice with 3 -body interactions on the triangles, namely, Boltzmann weights $\quad\left(w_{1}, w_{2}, w_{3}\right)=\left(q+3 v+3 v^{2}+v^{3}, q+3 v+\right.$ $\left.v^{2}, q+3 v\right)$ according as the triangle has 1,2 , or 3 distinct spin values [19]. We then apply a Peierls argument to this general triangular-lattice model and prove that there exists ferromagnetic long-range order in an open region of $\left(w_{2} / w_{1}, w_{3} / w_{1}\right)$ space that includes the point $\left(w_{2} / w_{1}, w_{3} / w_{1}\right)=(1 / 2,0)$ corresponding to $q=3$ at zero temperature $(v=-1)$.

Choose a large box $\Lambda$ and fix all boundary spins (on the triangular sublattice) in the same state. Given a spin configuration on the triangular lattice, draw Peierls contours on the dual hexagonal lattice in the usual way, i.e., separating unequal spin values on the triangular lattice. We thus obtain a spanning subgraph of the hexagonal lattice in which the vertices have degree 0,2 , or 3 according as the corresponding triangle has 1,2 , or 3 distinct spin values.

Consider first the case $w_{3}=0$ (this covers the zerotemperature 3-state diced-lattice model, i.e., $q=3$ and $v=-1$ ). Then the Peierls contours have no vertices of degree 3, so they are disjoint unions of self-avoiding polygons (SAPs) on the hexagonal lattice. Each contour edge gets a weight $w_{2} / w_{1}$, and each contour gets an additional weight $q-1$ to count the possible values for the spin change modulo $q$ when crossing the contour. If the probability of having at least one contour is less than $(q-1) / q$, then the spin at the origin has probability greater than $1 / q$ of being in the same state as the boundary condition, hence there is long-range order. This occurs whenever

$$
\sum_{n=6}^{\infty} q_{n}^{(1)}\left(w_{2} / w_{1}\right)^{n}<1 / q,
$$

where $q_{n}^{(1)}$ is the number of $n$-step hexagonal-lattice SAPs surrounding the origin of the triangular lattice, or equivalently the first area-weighted moment for $n$-step hexagonal-lattice SAPs modulo translation. To bound this sum, we use the exact values of $q_{n}^{(1)}$ for $6 \leq n \leq 140$ [20] and the bound $q_{n}^{(1)} \leq\left(n^{2} / 36\right) 1.868832^{n-2}$ for even $n \geq$ 142 [21]. For $q=3$ we deduce long-range order whenever $w_{2} / w_{1}<0.503417$, which barely includes the desired value $w_{2} / w_{1}=1 / 2$ [22].

The case $w_{3}>0$ is more complicated because of the presence of degree-3 vertices, but it can be shown [23] that 
if the Peierls inequality holds at a given value of $w_{2} / w_{1}$ when $w_{3}=0$, then it will also hold at that same value of $w_{2} / w_{1}$ whenever $w_{3} / w_{1}$ is sufficiently small. This proves that the 3-state diced-lattice antiferromagnet has longrange order on the triangular sublattice at all sufficiently low temperatures. With a little more work [23], we can prove antiferromagnetic long-range order on the whole diced lattice.

Monte Carlo simulation. - We simulated the dicedlattice Potts antiferromagnets for $q=2,3,4$ on $L \times L$ lattices ( $3 \leq L \leq 768$ ) with periodic boundary conditions, using the Wang-Swendsen-Kotecký (WSK) cluster algorithm [24]. Since the diced lattice is bipartite, the WSK algorithm is guaranteed to be ergodic $[10,25]$ and there is reason to hope that critical slowing-down might be absent (as for the square lattice [25]) or at least small.

We measured the energy $\mathcal{E}$, the sublattice magnetizations $\mathcal{M}_{\text {hex }}$ and $\mathcal{M}_{\text {tri }}$, and the second-moment correlation length $\xi$ [26]. We focused attention on the Binder-type ratio $R=\left\langle\mathcal{M}_{\text {stagg }}^{4}\right\rangle /\left\langle\mathcal{M}_{\text {stagg }}^{2}\right\rangle^{2}$ where $\mathcal{M}_{\text {stagg }}=\mathcal{M}_{\text {tri }}-$ $\mathcal{M}_{\text {hex }}$, which tends in the infinite-volume limit to $(q+$ 1) $/(q-1)$ in a disordered phase and to 1 in an ordered phase, and is therefore diagnostic of a phase transition. The ratio $\xi / L$ plays a similar role. Finally, we studied $\left\langle\mathcal{M}_{\text {stagg }}^{2}\right\rangle$ in order to estimate the leading magnetic critical exponent.

The Ising $(q=2)$ data confirm the exact solution [7]. For $q=4$, we find a finite correlation length uniformly down to zero temperature, with $\xi(v) \uparrow \approx 1.85$ as $v \downarrow-1$.

For $q=3$, a plot of $R$ versus $v$ for lattice sizes $3 \leq L \leq$ 96 is shown in Fig. 2, and shows a clear order-disorder transition at $v_{c} \approx-0.86$. Finer plots of $R$ and $\xi / L$ near the transition, for $48 \leq L \leq 768$, are shown in Figs. 3 and 4. We fit the data to Ansätze obtained from

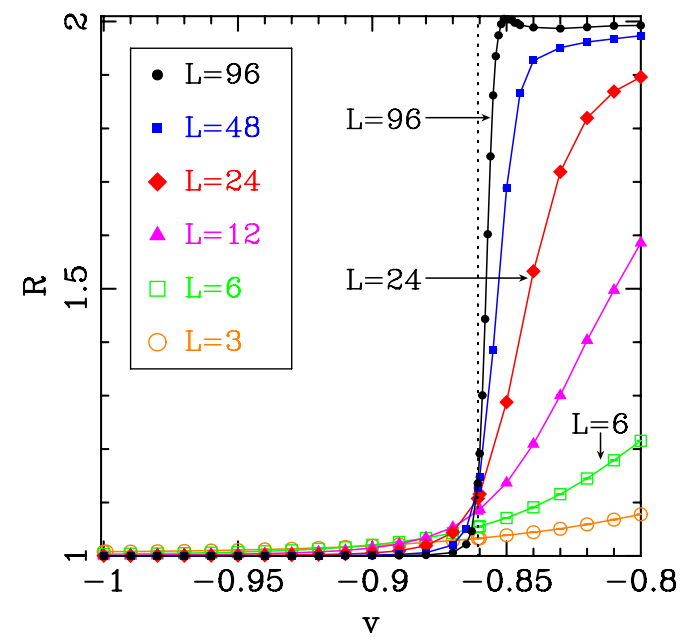

FIG. 2 (color online). Coarse plot for the Binder ratio $R$. Dotted vertical line marks the critical point predicted in $[17,18]$. Curves are straight lines connecting points, meant only to guide the eye.

$$
\begin{aligned}
\mathcal{O}= & \mathcal{O}_{c}+a_{1}\left(v-v_{c}\right) L^{1 / \nu}+a_{2}\left(v-v_{c}\right)^{2} L^{2 / \nu} \\
& +b_{1} L^{-\omega_{1}}+\cdots
\end{aligned}
$$

by omitting various subsets of terms, and we systematically varied $L_{\min }$ (the smallest $L$ value included in the fit). We also made analogous fits for $\left\langle\mathcal{M}_{\text {stagg }}^{2}\right\rangle / L^{\gamma / \nu}$. Comparing all these fits, we estimate the critical point $v_{c}=$ $-0.860599 \pm 0.000004$, the critical exponents $\nu=$ $0.81 \pm 0.02$ and $\gamma / \nu=1.737 \pm 0.004$, and the universal amplitude ratios $R_{c}=1.170 \pm 0.007$ and $(\xi / L)_{c}=$ $0.995 \pm 0.007(68 \%$ subjective confidence intervals, including both statistical error and estimated systematic error due to unincluded corrections to scaling). These exponents are in excellent agreement with the values for the 3-state Potts ferromagnet: $\nu=5 / 6 \approx 0.833, \gamma / \nu=26 / 15 \approx$ 1.733 , and $R_{c}=1.1711 \pm 0.0005$ [27]. This confirms our expectation that the 3-body-interacting triangular-lattice ferromagnet, obtained by integrating out the hexagonal sublattice, lies in the universality class of the 3-state Potts ferromagnet.

For the triangular-sublattice spontaneous magnetization $M_{0}$, defined by $M_{0}^{2}=\lim _{L \rightarrow \infty}\left\langle\mathcal{M}_{\text {tri }}^{2}\right\rangle / V_{\text {tri }}^{2}$, we find $M_{0}=$ $0.936395 \pm 0.000006$ at $v=-1$, which is not far from the Peierls bound $M_{0} \geq 0.90497$ [28] and the heuristic estimates $M_{0} \approx 125 / 128 \approx 0.97656$ and $M_{0} \approx 21 / 22 \approx$ 0.95455 [23].

Details of the simulations will be reported later [23].

Final remarks. - Here is another construction that produces bipartite planar lattices (not, however, plane quadrangulations) with $q_{c}>3$ and indeed with $q_{c}$ arbitrarily large. Let $\mathcal{L}$ be any lattice, and let $\mathcal{L}_{2}$ be the lattice obtained from $\mathcal{L}$ by subdividing each edge into two edges in series. Then the Potts series law $\left(v_{1}, v_{2}\right) \mapsto v_{1} v_{2} /(q+$ $v_{1}+v_{2}$ ) [29] implies that the Potts model on $\mathcal{L}_{2}$ has a phase transition whenever $v^{2} /(q+2 v)=v_{\text {crit,ferro, } \mathcal{L}}(q)$. In particular, if $v_{\text {crit,ferro, } \mathcal{L}}(q)<1 /(q-2)$, then there is a

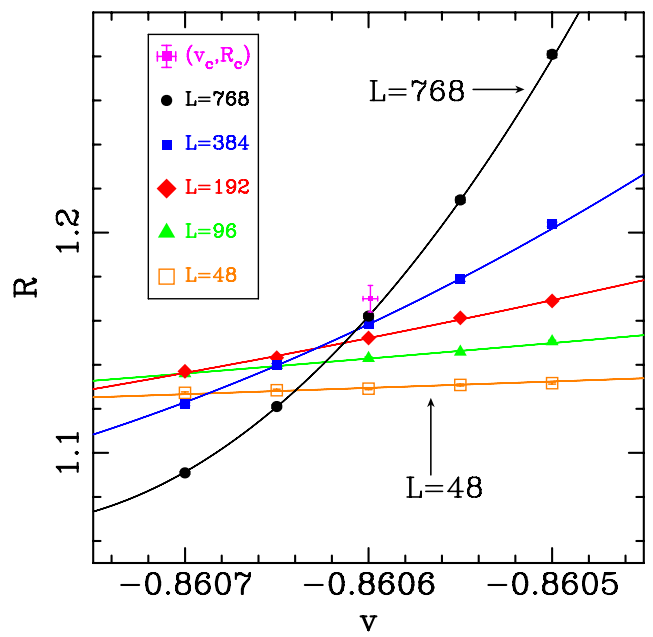

FIG. 3 (color online). Fine plot for the Binder ratio $R$. Curves are our fits to (2). Symbol indicates estimates of $v_{c}$ and $R_{c}$. 


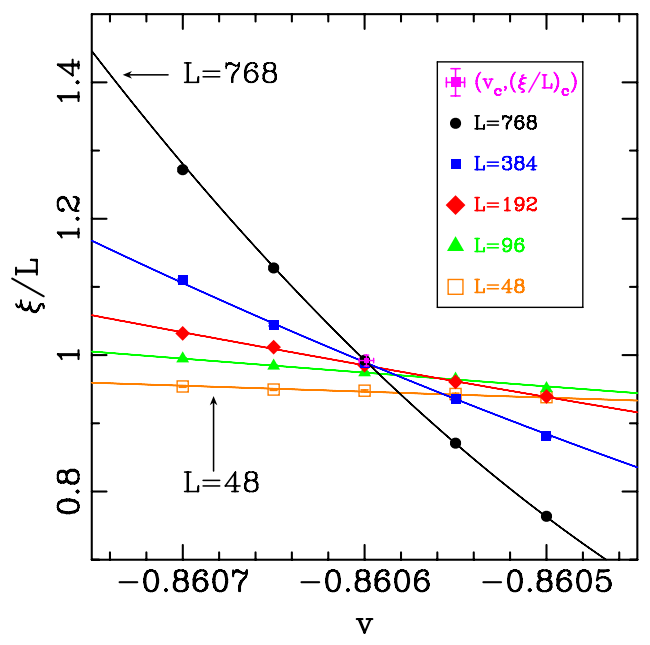

FIG. 4 (color online). Fine plot for $\xi / L$. Curves are our fits to (2). Symbol indicates estimates of $v_{c}$ and $(\xi / L)_{c}$.

solution $v \in(-1,0)$, so that $q_{c}\left(\mathcal{L}_{2}\right)>q$. For instance, the triangular lattice $\mathcal{T}$ has a ferromagnetic critical point when $v^{3}+3 v^{2}-q=0$, from which we conclude that $q_{c}\left(\mathcal{T}_{2}\right) \approx 3.117689$. Furthermore, Wierman [30] has constructed periodic plane triangulations $T(k)$ (obtained by subdividing triangles in the triangular lattice) whose bond percolation thresholds tend to zero as $k \rightarrow \infty$; and the Potts series-parallel laws show, more generally, that for each $q \geq 1$ one has $\lim _{k \rightarrow \infty} v_{\text {crit,ferro, } T(k)}(q)=0$ [31]. It follows that $\lim _{k \rightarrow \infty} q_{c}\left(T(k)_{2}\right)=+\infty$.

We thank Chris Henley, Neal Madras, and Gordon Slade for very helpful correspondence. We especially thank Cris Moore for discussions some years ago concerning the Peierls argument for Potts antiferromagnets; he independently suggested to consider the diced lattice. This work was supported in part by NSF Grant No. PHY-0424082, Spanish MEC Grants No. MTM2005-08618 and No. FIS2004-03767, and Czech Grants No. GAČR 201/ 06/1323 and No. MSM 0021620845. We thank the Isaac Newton Institute at the University of Cambridge, where this work was completed.

[1] R. B. Potts, Proc. Cambridge Philos. Soc. 48, 106 (1952).

[2] F. Y. Wu, Rev. Mod. Phys. 54, 235 (1982); 55, 315(E) (1983); F. Y. Wu, J. Appl. Phys. 55, 2421 (1984).

[3] R. J. Baxter, Exactly Solved Models in Statistical Mechanics (Academic, London, 1982).

[4] B. Nienhuis, J. Stat. Phys. 34, 731 (1984).

[5] P. Di Francesco, P. Mathieu, and D. Sénéchal, Conformal Field Theory (Springer-Verlag, New York, 1997).

[6] Some exceptions are the Ising model $(q=2)$ on the triangular lattice $\left(q_{c}=4\right)$, which has a zero-temperature critical point [J. Stephenson, J. Math. Phys. (N.Y.) 5, 1009 (1964)], and the Ising model on the kagome lattice $\left(q_{c}=\right.$ $3)$, which is noncritical at all temperatures, including zero temperature [7].
[7] I. Syozi, in Phase Transitions and Critical Phenomena, edited by C. Domb and M. S. Green (Academic, London, 1972), Vol. 1.

[8] See J. Salas and A. D. Sokal, J. Stat. Phys. 92, 729 (1998), and the references cited there.

[9] Some exceptions are the constrained square-lattice 4-state antiferromagnetic Potts model [10] and the triangularlattice antiferromagnetic spin-s Ising model for large enough $s$ [C. Zeng and C.L. Henley, Phys. Rev. B 55, 14935 (1997)], both of which appear to lie in a noncritical ordered phase at zero temperature.

[10] J. K. Burton, Jr. and C.L. Henley, J. Phys. A 30, 8385 (1997).

[11] M. den Nijs, M.P. Nightingale, and M. Schick, Phys. Rev. B 26, 2490 (1982)

[12] J. Kolafa, J. Phys. A 17, L777 (1984).

[13] D. A. Huse and A. D. Rutenberg, Phys. Rev. B 45, 7536 (1992).

[14] J. Kondev and C. L. Henley, Nucl. Phys. B464, 540 (1996).

[15] C. Moore and M.E. J. Newman, J. Stat. Phys. 99, 629 (2000).

[16] J. Kondev and C. L. Henley, Phys. Rev. B 52, 6628 (1995).

[17] I. Jensen, A. J. Guttmann, and I. G. Enting, J. Phys. A 30, 8067 (1997).

[18] H. Feldmann, R. Shrock, and S.-H. Tsai, Phys. Rev. E 57, 1335 (1998).

[19] The idea of integrating out one sublattice goes back at least to R. Kotecký, Phys. Rev. B 31, 3088 (1985).

[20] I. Jensen, J. Phys.: Conf. Ser. 42, 163 (2006); http:// www.ms.unimelb.edu.au/ iwan/polygons/Polygons_ser .html.

[21] This bound combines the isoperimetric inequality $q_{n}^{(1)} \leq$ $\left(n^{2} / 36\right) q_{n}$ (where $q_{n}$ is the number of $n$-step hexagonallattice SAPs modulo translation), the elementary bound $q_{n} \leq \mu^{n-2}$ (which follows from the supermultiplicativity $q_{m+n-2} \geq q_{m} q_{n}$, provable by concatenation), and the bound $\mu<1.868832$ [S.E. Alm and R. Parviainen, J. Phys. A 37, 549 (2004)].

[22] Most of the sum of this series comes from the crude estimate of the tail. If we use instead for $n \geq 142$ the estimated actual behavior $q_{n}^{(1)} \approx(1 / 4 \pi)(2+\sqrt{2})^{n / 2} n^{-1}$ [20], the condition (1) holds for $w_{2} / w_{1} \lesssim 0.541180$.

[23] R. Kotecký, J. Salas, and A. D. Sokal (to be published).

[24] J.-S. Wang, R. H. Swendsen, and R. Kotecký, Phys. Rev. Lett. 63, 109 (1989); Phys. Rev. B 42, 2465 (1990).

[25] S. J. Ferreira and A. D. Sokal, J. Stat. Phys. 96, 461 (1999).

[26] $\xi$ is an antiferromagnetic analogue of [8], Eq. (3.11).

[27] J. Salas and A. D. Sokal, J. Stat. Phys. 87, 1 (1997); T. M. Garoni, G. Ossola, M. Polin, and A.D. Sokal (to be published).

[28] This bound is based on the estimated actual behavior $q_{n}^{(1)} \approx(1 / 4 \pi)(2+\sqrt{2})^{n / 2} n^{-1}$ [20] for $n \geq 142$.

[29] A. D. Sokal, in Surveys in Combinatorics, 2005, edited by B.S. Webb (Cambridge University Press, Cambridge, England, 2005).

[30] J. C. Wierman, J. Phys. A 35, 959 (2002).

[31] The passage from Wierman's $G(k)$ to $T(k)$ uses the Fortuin-Kasteleyn-Ginibre (FKG) inequality, which needs $q \geq 1$. 